\title{
Topical issue on lattice field theory methods in hadron and nuclear physics
}

\author{
Published online: 17 July 2013 - (c) Società Italiana di Fisica / Springer-Verlag 2013
}

Since the first application of numerical Monte Carlo simulations in the late 1970s, lattice QCD has been steadily increasing in importance and applicability until it is now generally acknowledged as perhaps the most systematic and controlled way of seeking answers to important questions in strong interaction physics. Today's lattice practitioners can devote multi-teraflop computing resources to problems as a matter of routine, and are on the threshold of access to the petascale. However, innovations in algorithms for generating gauge ensembles, efficient sampling techniques, as well as conceptual progress in lattice actions and many other technical improvements have been equally important for achieving the current tight control over the necessary continuum, chiral and thermodynamic limits.

The calculation of the spectrum of the lowest-lying mesons and baryons, once considered the "Holy Grail" of the field, has now been performed with physically light quark masses. The impressive agreement between the experimentally determined masses and the corresponding lattice estimates has not only served to validate QCD as the theory of the strong interaction, but also given enormous credibility to the lattice approach. However, the ultimate goal of lattice QCD calculations is to make predictions for experiments and to provide accurate input for phenomenology. In the area of flavour physics this has largely been achieved, as accurate results for mesonic decay constants, form factors and mixing parameters, which serve to constrain the elements of the Cabibbo-Kobayashi-Maskawa matrix, have been published. Similarly, lattice QCD has had a major impact on the determination of the fundamental parameters of the Standard Model, by providing precise estimates for the masses of the light quarks and the strong coupling constant. These successes have established lattice QCD as a mature field, and this status is further underlined by the fact that an international collaboration ${ }^{1}$ is now preparing global averages of lattice results, very much in the spirit of the Particle Data Group. Lattice QCD calculations have also had a major impact on the experimental programme in heavy ion collisions, for instance, by providing accurate estimates for the temperature which marks the transition from conventional hadronic matter to a plasma-like phase.

Lattice studies of the strong interaction are also facing new challenges. While the spectrum of ground-state mesons and baryons has been confirmed, the focus of current studies is shifting to the much more difficult determination of the excitation spectrum. Lattice calculations of the energy levels of quarkonia and baryon resonances, including their widths, already play an important role for the physics programme at future facilities, such as FAIR at Darmstadt or the planned experiments at Jefferson Lab following the $12 \mathrm{GeV}$ upgrade. Other topics which have been tackled recently include the $a b$ initio treatment of isospin breaking and the determination of the hadronic corrections to the muon $(g-2)$. In both cases, lattice simulations still face significant technical difficulties, perhaps requiring enormous computational resources.

Moreover, within this general progress, lattice methods are beginning to pervade the domain of nuclear physics. Here the main requirements are the treatment of residual interactons between color-singlet hadrons, either pairwise or in some complicated multi-hadron system. Eventually, one would like to attain a quantitative treatment of QCD thermodynamics at large baryon densities, appropriate for describing the conditions in relativistic ion collisions or the cold bulk matter found in neutron stars. Attention is evolving from the determination of critical parameters and the thermodynamic equation of state to include information about the excitation spectrum and transport in the hot or dense medium. Monte Carlo simulations of effective theories for multi-hadron systems have also proved extremely valuable for studying the properties of light nuclei, such as ${ }^{12} \mathrm{C}$. In many cases the questions addressed predate QCD itself.

\footnotetext{
1 http://itpwiki.unibe.ch/flag/index.php
} 
It is truly remarkable that almost 30 years after Kenneth Wilson's seminal paper on the formulation of QCD on a Euclidean space-time grid, numerical simulations have become a versatile tool for studying a wide range of problems and phenomena in particle, hadron and nuclear physics. The articles collected in this Topical Issue are focussed on current challenges for lattice QCD in the areas of hadron phenomenology, nuclear physics and QCD thermodynamics. They have been commissioned from some of the leading experts in the field, who have developed new methods to explore these issues.

The first three contributions deal with multi-hadron systems and topics in nuclear physics. The review by Aoki introduces a method (often dubbed the HAL QCD approach) for treating nucleon-nucleon interactions, based on determining the $(N N)$ potential in a first step, which is then used as input for solving a Schrödinger equation. In this way observables such as the $(N N)$ scattering phase shifts can be determined. The crucial quantity in this formalism is the $(N N)$ Nambu-Bethe-Salpeter wave function which must be extracted from suitable correlation functions computed in a lattice simulation.

Epelbaum, Krebs, Lähde, Lee and Meißner study a fundamental question in nuclear astrophysics, namely the production rates of carbon and oxygen in red giant stars, which have wide-ranging consequences for the evolution of organic life. To this end they employ numerical simulations of a discretised chiral effective theory for the nuclear potential and determine how the energy levels of light nuclei vary as a function of the light quark masses. Their work is a fascinating example of how lattice simulations allow for the treatment of nuclear reactions and their implications for long-standing problems in nuclear physics, such as the properties of the Hoyle state.

The third article in this area, contributed by Detmold, gives an overview of several complementary approaches to multi-hadron systems. In a seminal paper by Lüscher it was demonstrated that many properties of multi-hadron states, such as scattering phase shifts, can be accurately and elegantly determined by computing their energy levels in a finite volume. Detmold's review is focussed on calculations of binding energies of multi-meson and multi-baryon systems, including the $H$-dibaryon, but also systems with baryon number $B>2$. It is clear from this review that the required computational resources are quite enormous, if the treatment of multi-hadron systems is based directly on QCD. In order to be able to tackle larger nuclei calls for new methods which reduce the overall numerical cost.

The article by Meyer leaves the domain of nuclear physics and addresses an important process in hadron phenomenology, i.e. the decay amplitude for the process $\pi^{0} \rightarrow \gamma \gamma$. The particular interest is driven by the problem of the muonic $(g-2)$ and, more specifically, the contribution due to hadronic light-by-light scattering. Meyer's analysis has no explicit reference to a lattice cutoff, but tries to apply the well-known relation between the discrete spectrum on a torus and scattering phase shifts among final-state particles to this particular decay process.

The article by Bazavov and Petreczky switches gear by considering an old problem, the interaction between static color sources, but now with a non-zero temperature $T$ both above and below the critical $T_{c}$ associated with the quark-hadron thermal phase transition. With $T>0$ there are contributions to correlation functions from so-called "hybrid potentials", with the result that effective mass plots no longer exhibit the plateau behaviour familiar from spectroscopy at $T=0$; this paper forms a nice introduction to the ensuing issues.

When considering QCD as a thermal system the goal is the elucidation of the phase diagram in the $(\mu, T)$ plane, where $\mu$ is the baryochemical potential (other refinements under consideration include axes for isospin chemical potential, or external magnetic field). Differing regions of the plot present theoretical challenges akin to strongly interacting atomic physics (low $T$, low $\mu$ ), plasma physics (high $T$, low $\mu$ ), or condensed matter physics (low $T$, high $\mu$ ). All are legitimate foci of study in nuclear physics. The principal barrier to applying lattice QCD in the interior of the phase diagram where $\mu \neq 0$ is that in Euclidean metric the action $S(\mu)=S^{*}(-\mu)$, implying that importance sampling based on a positive definite probability measure, the basis for Monte Carlo "simulation", is effectively inoperable once $\mu \neq 0$ renders $S$ complex-valued.

In recent years moderate progress has been possible in the plasma physics regime $\mu / T \lesssim 1$, either by reweighting from ensembles generated with real-valued actions, by Taylor expansion in even powers $(\mu / T)^{2 n}$, or by analytically continuing from simulations perfomed with $\mu$ pure imaginary. The paper of Ejiri continues in this tradition, tackling the phenomenologically important question of the order of the quark-hadron phase transition as a function of both quark mass and $\mu$, on the assumption that the phase fluctuations of $S$ can be modelled by a Gaussian distribution. By contrast, it is possible to evade the so-called sign problem completely by considering a world with not three but two colors, with gauge group $\mathrm{SU}(2)$ and baryons formed from diquark bosons. The contribution of Boz, Cotter, Fister, Mehta and Skullerud is the latest in a series applying orthodox simulation techniques to this system; the main result is that cold dense matter seems to be simultaneously degenerate (implying a quark Fermi surface), and confined. The current paper concentrates on gluonic observables, which only depend on $\mu$ via virtual quark loops, in particular those multiply wound around Euclidean time.

An area of theoretical physics with very definite nuclear origins is the study of random matrices and their eigenspectra. The techniques developed in this arena have recently found a beautiful application in the study of QCD in the so-called mesoscopic regime, and many consequent exact results for the low-energy spectrum of the Dirac operator have been corroborated by numerical LQCD. The same techniques are here applied by Kanazawa, Wettig and Yamamoto to the ground state of QCD-like theories with no sign problem, and a relation for the energy gap $\Delta$ of a BCS-paired state in terms of the spectral density function is derived. 
The final two contributions both outline possible solutions to the sign problem applicable to QCD itself. This is an area of high risk and still higher reward; success will not only unlock high-density baryonic matter, but also many other important problems in condensed matter and many-body physics, an example being cuprate superconductivity. The paper of Aarts, Bongiovanni, Seiler, Sexty and Stamatescu reviews recent progress in applying Langevin dynamics to models with complex action. In this approach field variables are complexified and evolved through a fictitious time with both a drift force depending on $S$ and a stochastic force component, with the idea that quantum fluctuations are thus modelled in the ergodic limit. Configuration space is thus sampled in a completely different manner to Monte Carlo, and experience of how to control the calculations and when the results can be trusted is gradually developing; here a recent development, gauge cooling is described and tested for the first time. The contribution of Chandrasekharan employs a completely different strategy, this time reformulating the problem in terms of variables which can be regrouped to completely remove sign problems. This approach has many advantages: it permits efficient simulation in the chiral limit quark mass $m=0$, technically challenging for orthodox hybrid Monte Carlo; and it has already achieved impressive results for quantum critical points in low-dimensional fermionic systems.

Overall, then, this is a lively and fertile time for applying lattice technology to nuclear physics problems, and it is surely appropriate to publicise these efforts to a wider audience. We thank all the contributors for their enthusiasm and hard work making this volume possible, and the Editorial Board and publishers of The European Physical Journal A for their support and encouragement.

Simon Hands and Hartmut Wittig

Guest Editors 